\section{CPS-242 REGULATORY VALIDATION OF PRESCRIPTIONS AT A TEACHING HOSPITAL}

${ }^{1} \mathrm{M}$ Mseddi ${ }^{*},{ }^{1} \mathrm{~K}$ Myriam, ${ }^{1} \mathrm{~A}$ Hamdi, ${ }^{2} \mathrm{~T}$ Manel. ${ }^{1}$ Faculty of Pharmacy- University of Monastir, Pharmacology Department, Monastir, Tunisia; ${ }^{2}$ Teaching Hospital Hedi Chaker, Pharmacy Department, Sfax, Tunisia

10.1136/ejhpharm-2019-eahpconf.391

Background Regulatory compliance of prescriptions is a key aspect of good performance of pharmaceutical validation within the hospital. Several irregularities have been detected in daily practice.

Purpose Estimate the nature and prevalence of irregularities in prescriptions at a teaching hospital's pharmacy in order to establish an action plan.

Material and methods A one-day study (October 2017) conducted on all (out/in) patients' prescriptions at the teaching hospital. The patient registration number was verified systematically via a dedicated software ADMIS.

The quantitative and qualitative analysis of prescriptions was done via SPSS software v23. The analysis of multivariate data was made by Kiviat Diagrams.

Results The analysis was based on 590 prescriptions, of which 393 were from the external pharmacy and 197 from hospitalised patients.

The prescriptions were completely delivered in $80 \%$ of the cases. Ninety-five per cent of the validated prescriptions pharmaceutically were from the hospitalised services against $73.7 \%$ for prescriptions resulting from the consulting services.

All the prescriptions bore the correct registration number corresponding to the right patient, and treatment duration was present in $100 \%$ of the cases.

The non-conformity (absence/illegibility) was due to the prescriber's stamp in $11.9 \%$, the date of prescription in $3.1 \%$ of cases and the seal of the service in $7.5 \%$ of all cases corresponding to $11 \%$ of outpatients.

The origin of the non-compliance issued mainly from the haematology department (11.4\%), followed by cardiology $(10.5 \%)$ and endocrinology $(8.8 \%)$, and in $25 \%$ of cases the service was unidentifiable.

This critical analysis of the regulatory aspect allowed us to identify several causes requiring a plan of action, mainly: nonavailability of the stamps of some freshly graduated doctors; the ink was not available; superimposition of service and prescribing stamps; and forgetting the stamp on the prescription.

Consequently, official letters were sent to all physicians reminding them about regulatory requirements for medical prescribing. An evaluation is planned within a year.

Conclusion Regulatory validation of prescriptions is a preliminary and essential step in pharmaceutical validation. A critical analysis of the irregularities makes it possible to establish a plan of action with specific procedures and proves periodically necessary as an indicator of the good functioning of the system.

\section{REFERENCES AND/OR ACKNOWLEDGEMENTS}

No conflict of interest.

\section{CPS-243 EFFECTIVENESS AND USE OF OFF-LABEL TREATMENTS IN A GENERAL HOSPITAL}

A Murgadella Sancho*, B Gracia Garcia, A Morales Triado, N San Juan Martínez. Hospital Moisès Broggi, Pharmacy, Sant Joan Despi, Spain

10.1136/ejhpharm-2019-eahpconf.392
Background The off-label use of drugs is common in a hospital setting. However, some of these treatments have low scientific evidence.

Purpose The study aim was to describe the off-label use of drugs in the hospital and to assess the effectiveness of these treatments. Material and methods We revised the authorised off-label applications between January 2016 and July 2018. We excluded all the off-label oncology treatments.

Clinical history, date of application, medical service, drug, indication and symptomatic improvement of disease were collected.

We considered effectiveness when the patient experienced improvement in most symptoms related to the disease (total effectiveness) or improvement in some symptoms (partial effectiveness). When the drug was not given for any reason, or the treatment was not finished for toxicity, it was considered not assessable.

Results A total of 84 applications were analysed. The evolution of these was: 32 applications in 2016, 27 in 2017 and 25 in 2018.

The medical services were: neurology (20\%), nephrology $(17 \%)$, nigestive $(15 \%)$, ophthalmology $(14 \%)$, otorhinolaryngology $(6 \%)$ and other services (28\%).

The most demanded drugs were rituximab $(27 \%, n=23)$, botulinum toxin A $(20 \%, n=17)$ and human immunoglobulin $(18 \%, \mathrm{n}=15)$.

The indications for rituximab were: membranous nephropathy $(n=5)$, systemic lupus erythematosus $(n=4)$, Sjögren syndrome $(n=2)$, cryoglobulinemic vasculitis $(n=2)$ and others $(n=10)$.

The indications for botulinum toxin A were: achalasia $(n=13)$, spasmodic dysphonia $(n=3)$ and Frey syndrome $(n=1)$.

The indications for human immunoglobulins were: myasthenic crisis $(n=7)$, autoimmune encephalitis $(n=3)$ and other indications $(\mathrm{n}=5)$.

Of all applications $(\mathrm{n}=84), 15$ were not assessable: 10 because the treatment was not administrated and five because of its toxicity.

From all patients with an assessable treatment $(\mathrm{n}=69), 70 \%$ $(\mathrm{n}=48)$ experienced symptomatic improvement of the disease: in $48 \%(\mathrm{n}=23)$ the treatment was totally effective and in $52 \%(n=25)$ it was partially effective.

Conclusion There is a high variability in the off-label use of drugs. It is necessary to develop protocols to unify the criteria of use of the most common treatments.

Despite low-level published evidence, the off-label treatments were effective in most patients, so they suppose a benefit for patients with few therapeutic options.

\section{REFERENCES AND/OR ACKNOWLEDGEMENTS}

http://www.elsevier.es/es-revista-revista-calidad-asistencial-256pdf-S1134282X12000760

https://www.sefh.es/fh/118_121v35n05pdf009.pdf

No conflict of interest.

\section{CPS-244 TIME TO PERFORM MEDICATION RECONCILIATION AT ADMISSION IN A NEUROLOGY UNIT: COMPARISON BETWEEN PROACTIVE AND RETROACTIVE PROCESSES}

${ }^{1} \mathrm{C}$ Dubrou, ${ }^{1} \mathrm{~V}$ Nail*, ${ }^{2} \mathrm{~T}$ Horowitz, ${ }^{2} \mathrm{~V}$ Mira, ${ }^{2} \mathrm{JP}$ Azulay, ${ }^{1} \mathrm{G}$ Hache. ${ }^{1}$ University Hospitals of Marseille, Pharmacy, Marseille, France; ${ }^{2}$ University Hospitals of Marseille, Neurology, Marseille, France

10.1136/ejhpharm-2019-eahpconf.393

Background Medication reconciliation (MR) at admission is a multidisciplinary process which aims to ensure hospital 